\title{
1 A Statistical Physics Approach for Disease Module Detection
}

2 Xu-Wen Wang ${ }^{1}$, Dandi Qiao ${ }^{1}$, Michael Cho ${ }^{1}$, Dawn L. DeMeo ${ }^{1}$, Edwin K. Silverman ${ }^{1}$, Yang-Yu

$3 \mathrm{Liu}^{1}$

$4{ }^{I}$ Channing Division of Network Medicine, Department of Medicine, Brigham and Women's

5 Hospital and Harvard Medical School, Boston, Massachusetts 02115, USA

\section{ABSTRACT}

Extensive evidence indicates that the pathobiological processes of a complex disease are 9 associated with perturbation within specific disease neighborhoods of the human protein-protein 10 interaction (PPI) network (a.k.a. the interactome), often referred to as the disease module. Many 11 computational methods have been developed to integrate the interactome and omics profiles to 12 extract context-dependent disease modules. Yet, existing methods all have fundamental limitations 13 in terms of rigor and/or efficiency. Here, we developed a statistical physics approach based on the 14 random-field Ising model (RFIM) for disease module detection, which is both mathematically 15 rigorous and computationally efficient. We applied our RFIM approach with genome-wide 16 association studies (GWAS) of six complex diseases to examine its performance for disease 17 module detection. We found that our RFIM approach outperforms existing methods in terms of 18 computational efficiency, connectivity of disease modules, and robustness to the interactome 19 incompleteness. 


\section{INTRODUCTION}

Complex diseases are believed to be caused, among other factors, by combinations of genetic alterations affecting cellular systems ${ }^{1}$. Integration of disease-related genomics, epigenomics, transcriptomics and other omics data with the human protein-protein interaction network (or interactome) could provide insight into the molecular mechanisms of complex diseases. A disease network module represents a group of cellular components (e.g., dysfunctional pathways, complexes or crosstalk subgraphs between pathways in a molecular interaction network) that together contribute to normal cellular function that, when disrupted, may produce a particular disease phenotype ${ }^{1}$. The concept of the disease module is based on the local hypothesis that if a gene or molecule is involved in a specific biochemical process or disease, its direct interactors might also be suspected to play some role in the same biochemical process or disease ${ }^{2-4}$. For example, proteins that are involved in the same disease show a high propensity to interact with each other ${ }^{5,6}$, and genes that are mutated in diseases with similar phenotypes have a significantly increased tendency to interact directly with each other ${ }^{7,8}$. Hence, we expect that each disease can be linked to a well-defined neighborhood of the cellular molecular interactome, i.e., a disease module, which is likely to include multiple disease-modifying genes that mediate various epigenetic, transcriptional and post-translational phenomena. Detection of the disease module for a pathophenotype of interest in turn can guide further experimental work towards uncovering the disease mechanism, predicting disease genes and informing drug development ${ }^{9-13}$.

There are many existing methods for disease module detection ${ }^{14-16}$. For example, ModuleDiscoverer ${ }^{17}$ used a heuristic-based approximation of the community structure based on the maximal clique enumeration formalism. DIAMOnD ${ }^{18}$ starts from a set of seed genes and prioritizes the other proteins of the interactome for their putative disease relevance. However, those methods all have fundamental limitations. For example, seed-gene based methods heavily rely on the initial selection of seed genes. As for heuristic-based methods that attempt to find optimal subgraphs within the molecular interaction network, they turn out to be computationally intractable in general.

To address the limitations of previous methods, here we develop a novel statistical physics approach for disease module detection based on the random-field Ising model (RFIM) ${ }^{19-21}$. We hypothesize that optimizing the "score" of the whole network rather than a particular subgraph will discover a biologically more meaningful disease module. Optimizing the score of the whole network (with appropriately assigned node weights and edge weights calculated from omics profiles) can be mapped to the ground state problem of RFIM and then solved exactly by the maxflow algorithm with polynomial time complexity ${ }^{22}$. The resulting disease module might not be a single connected component or a tree graph at all. Using this approach, all of the disease related subgraphs will emerge simultaneously.

To compare our RFIM approach with existing methods, we applied our RFIM approach and two representative methods to genome-wide association studies (GWAS) of six different complex diseases: asthma ${ }^{23}$, breast cancer ${ }^{23}$, chronic obstructive pulmonary disease ${ }^{24}$ (COPD), cardiovascular disease ${ }^{25}$ (CVD), diabetes ${ }^{26}$, and lung cancer ${ }^{23}$. All SNPs were annotated to the 
nearest genes and gene-wise p-values were obtained by using a known approximation of the sampling distribution using MAGMA ${ }^{27-29}$. These gene-wise p-values were further integrated with the human interactome to identify the disease module. We found that our RFIM approach displays high computational efficiency and superior scalability. Moreover, the disease module identified by RFIM is much denser and includes more disease-associated pathways than other methods. Finally, we demonstrated clear evidence that RFIM is less sensitive to the incompleteness of the interactome than other methods.

\section{METHOD}

We formalize the disease module detection problem as a statistical physics problem as follows. We represent the state of node (gene) $i$ by a binary variable $\sigma_{i}= \pm 1$, where +1 (or -1 ) means gene $i$ is "active" (or "inactive"), i.e., belongs to the disease module (or not), respectively. The disease module is captured by the state of all genes, denoted as $\left\{\sigma_{i}\right\}$. Given a graph $G=(V, E)$, i.e., the PPI network with a node set $V$ (with node weights $\left\{h_{i}\right\}$ ) and an edge set $E$ (with edge weights $\left\{J_{i j}\right\}$ ), we aim to find the optimal gene state $\left\{\sigma_{i}\right\}$, also called the ground state, that minimizes the following cost function:

$$
\mathcal{H}\left(\left\{\sigma_{i}\right\}\right)=-\sum_{(i, j) \in E} J_{i j} \sigma_{i} \sigma_{j}-\sum_{i \in V}\left(H+h_{i}\right) \sigma_{i} .
$$

The subgraph induced by the active nodes in the ground state is the disease module.

The first term in the right-hand side of Eq. [1] represents the influence of nodes (genes) on their neighbors' states. By defining non-negative edge weights $\left\{J_{i j}\right\}$, we implicitly assume that neighboring nodes (genes) in the interactome tend to have the same state, i.e., they tend to belong to the disease module (or not) together. In other words, $J_{i j}>0$ favors positive correlations between the states of nodes $i$ and $j$. The stronger the $J_{i j}$ value, the more likely nodes $i$ and $j$ have the same state. In the case of $J_{i j}=0$, the states of nodes $i$ and $j$ are totally independent. The second term in the right-hand side of Eq. [1] stands for the intrinsic preference of nodes belonging to the disease module regardless of the states of their neighboring nodes. The intrinsic preference of node $i$ can be quantified as $h_{i}=\Phi^{-1}\left(1-p_{i}\right)$, where $\Phi^{-1}$ is the inverse normal cumulative distribution function and $p_{i}$ is the p-value of node (gene) $i$ calculated from the omics profile (see Fig.1a). Note that $h_{i}$ is a monotonically decreasing function of $p_{i}$ with $h_{i} \rightarrow+\infty$ as $p_{i} \rightarrow 0$ and $h_{i} \rightarrow-\infty$ as $p_{i} \rightarrow 1$. Nodes with very positive $h_{i}$ (or equivalently, very small $p_{i}$ ) tend to belong to the disease module.

The parameter $H$ is introduced to regulate the contributions of the two terms in Eq. [1]. Note that the value of $H$ effectively controls the average state variable $M \equiv 1 / N \sum_{i=1}^{N} \sigma_{i}$ of the system, or equivalently, the disease module size given by $(1+M) N / 2$ (see Fig.1b). For example, if $H$ is extremely low such that $\left(H+h_{i}\right)<0$ for all the nodes, then the ground state is simply $\left\{\sigma_{i}=-1\right\}$, i.e., all nodes will be inactive $(M=-1$ and there is no disease module, which is a trivial solution, Fig.1c). With gradually increasing $H$, more nodes will be active (Fig.1d). Eventually, if $H$ is high enough such that $\left(H+h_{i}\right)>0$ for all the nodes, then the ground state is 
$112\left\{\sigma_{i}=+1\right\}$, i.e., all nodes will be active $(M=+1$ and the whole interactome is the disease module,

113 which is also a trivial solution, Fig.1e). Hence, controlling the parameter $H$, we can obtain a 114 disease module with any desired size between 0 and $N$.

115 Note that Eq. [1] describes exactly the energy (or "Hamiltonian") of the RFIM rooted in 116 statistical physics, especially in the study of disordered magnets ${ }^{30}$. In its most general form, the 117 RFIM applies to an ensemble of magnetic particles interacting with each other in the presence of 118 an external magnetic field $H$. Each magnetic particle (also called spin) has two states $\sigma_{i}= \pm 1$ 119 (i.e., UP or DOWN). $h_{i}$ 's are local magnetic fields (due to impurities) acting on each spin and $120 h_{i}>0($ or $<0)$ favors UP (or DOWN) state of spin $i$, respectively. The coupling constants $J_{i j}>0$

121 capture the ferromagnetic interactions between spins $i$ and $j$ such that two spins next to each other 122 will tend to align to save the energy of the system. The control parameter $H$ is just the external 123 magnetic field, acting uniformly on all the spins to align them in the direction of $H$. The average 124 state variable (or order parameter) $M$ is the magnetization of the spin system.

125 In our calculation, we define the edge weight $J_{i j}=\max \left(0, h_{i}+h_{j}\right)$ if nodes $i$ and $j$ are 126 connected in the interactome, and $J_{i j}=0$ otherwise. This definition of $J_{i j}$ is to ensure that (1) the 127 two terms in the right-hand side of Eq. [1] are numerically comparable; (2) $J_{i j}$ is non-negative even 128 in case the intrinsic preference of nodes $i$ (or $j$ ) belonging to the disease module is extremely low 129 (i.e., with $h_{i}$ or $h_{j} \rightarrow-\infty$ ). Note that the disease module calculated from the active nodes in the 130 ground state of the RFIM is not necessarily a single connected component. In fact, it might contain 131 multiple connected components in the network $G$. To ensure the connectivity of the disease module 132 is not too low, we modify the intrinsic preference of node $i$ belonging to the disease module as $133 \tilde{h}_{i}=\max \left(h_{i}, \max _{j \in \partial i} h_{j}\right)$, where $\partial i$ represents the neighborhood (i.e., the set of neighboring nodes) of node $i$.

135 Our RFIM approach for the disease module detection has several advantages and novelties 136 over existing approaches: (1) Since the ground state and the $M(H)$ curve of the RFIM can be 137 exactly calculated with polynomial time complexity ${ }^{22,31}$, we can run the calculation on very large

138 data sets, e.g., human interactome, without any difficulty. (2) Both edge weights and node weights 139 are naturally considered. (3) The inferred disease module is not necessarily a single component or 140 a tree graph at all.

\section{RESULTS}

\section{Associations between gene significance and degree}

144 We considered gene-wise p-values calculated from the GWAS of six complex diseases: asthma ${ }^{23}$, 145 breast cancer ${ }^{23}$, chronic obstructive pulmonary disease ${ }^{32}$ (COPD), cardiovascular disease ${ }^{25}$ (CVD), 146 diabetes $^{26}$, and lung cancer ${ }^{23}$, respectively. For each disease, we overlaid the GWAS gene-wise p-

147 values onto the human interactome. For the human interactome, we used two well-established ones:

148 (1) STRING ${ }^{23}$; and (2) iRefIndex ${ }^{34,35}$. Before we identify the disease module, we examined the 149 association between the gene-wise p-value and the gene (node) degree in the interactome. We 150 found that those genes with low p-values (or high p-values) in GWAS typically have low degrees 
151 (or high-degree, i.e., hubs) in the interactome, respectively. Interestingly, this association is quite 152 robust to different diseases and interactomes (see Fig.2). We suspected that this association might 153 be related to the gene essentiality. For example, a previous study on the PPI of the yeast 154 Saccharomyces cerevisiae found that many hub genes are essential for survival of the cell, and 155 yeast cannot grow and multiply without them ${ }^{36}$. Thus, the SNPs annotated to the essential genes

156 are less likely to be functional variants. To validate this hypothesis, we used a reference of gene 157 essentiality constructed by genome-wide single-guide RNA library for genes required for 158 proliferation and survival in a human cancer cell line ${ }^{37}$. A CRISPR score is defined as the average $159 \log _{2}$ fold-change in the abundance of all single-guide RNA targeting a given gene and of the 18,166 160 genes, 1,878 scored as essential. We employed this reference to dichotomize genes in GWAS to 161 essential and non-essential. We found that the gene-wise p-values of essential genes in the GWAS 162 of asthma, CVD, and diabetes are significantly higher than that of non-essential genes (see 163 Fig.S1a). Moreover, we found that the degrees of those essential genes are significantly higher 164 than non-essential genes over all six diseases and regardless of the interactome analyzed here (see 165 Fig.S1b,c).

\section{Computational complexity of disease module detection}

168 After overlaying the GWAS gene-wise p-values onto the human interactome, we can identify the 169 disease module using various methods. For our RFIM approach, we calculated the $M(H)$ curve of 170 the RFIM by systematically tuning $H$. The disease module is detected from the ground state of the 171 RFIM at $H=H_{\mathrm{c}}$. We chose $H_{\mathrm{c}}$ to ensure the disease module reaches a desired size (i.e., consisting 172 of $5 \%$ of the total genes in the GWAS). In DIAMOnD, the most significantly connected node 173 (lowest $p$-value) is integrated into the module at each step of the iteration until the desired module 174 size is reached ${ }^{18}$ (and we chose the desired module size to be 500 for each disease). Given an 175 interactome, ModuleDiscoverer first approximates the underlying community structure by 176 iterative enumeration of gene cliques from random seed genes. Then, the union of all significantly 177 enriched cliques are ensembled into a large module ${ }^{17}$. We assessed the computational complexity 178 of different methods measured as the average computing time across all the six diseases. We found 179 that RFIM and DIAMOnD are the two most efficient methods, which require significantly shorter 180 running time than ModuleDiscoverer (see Fig.3). Moreover, we found that RFIM shows superior 181 scalability over other methods across different interactomes. This scalability is due to the fact that 182 the ground state of RFIM can be exactly solved with polynomial time complexity.

\section{Connectivity of the disease module}

185 By definition, the disease module is a specific neighborhood within the interactome. Hence, the 186 connectivity of the disease module should not be too low. We calculated the mean degree of the 187 disease module detected by each method, finding that the disease module identified by RFIM 188 shows the highest mean degree in all (or two of the six) diseases using the STRING (or iRefIndex) 189 interactome, respectively (see Fig.4a,b). Moreover, we found that the mean degree of disease 
module identified from RFIM is higher or comparative to the mean degree of the whole interactome for all the diseases analyzed in this work. Yet, the mean degree of disease modules identified by DIAMOnD (or ModuleDiscoverer) is much lower than that of the whole interactome for most diseases considered here.

\section{Pathway enrichment analysis}

To gain mechanistic insights into the list of genes in the disease module, we performed pathway enrichment analysis. In particular, we employed the ReactomePA package ${ }^{38}$ to obtain the enriched pathways for genes in the disease module with p-values lower than 0.05 cutoff adjusted by False Discovery Rate. Then, for each disease considered in this study, we extracted the diseaseassociated genes using the DisGeNET ${ }^{39}$ database and calculated the number of enriched pathways with at least two disease-associated genes. We found that the disease module calculated by RFIM shows the highest number of enriched pathways for five (six) diseases integrated with STRING (iRefIndex) in the disease module detection (see Fig.5) and this finding is valid if we counted the number of enriched pathways with at least four disease-associated genes (see Fig.S2).

\section{Robustness of disease module detection methods to the incompleteness of the interactome}

The phenotype-specific disease module is apparently determined by both interactome and genewise p-values. Yet, the interactome is highly incomplete, covering a small fraction of known PPIs. To investigate the sensitivity of each disease module detection method to the incompleteness of interactome, we calculated the overlap between the disease modules for different levels of incompleteness of the underlying interactome. We firstly applied the disease module into the original interactome. Then, a fraction of PPIs randomly selected from the original interactome were removed. Finally, we performed the disease module detection algorithms again on the perturbed interactome and compared the fraction of overlapping genes in the union genes of the disease module identified from the original and perturbed interactome, respectively. Fig. 6 shows that RFIM is more robust than other two methods, regardless of the fraction of PPIs have been removed.

\section{Disease module and enriched pathways}

We visualized the structure of the disease module identified for asthma using our RFIM approach and the STRING interactome. We found that there are several sub-modules, each of which includes at least one gene that is significantly associated with disease (based on GWAS gene-wise pvalue $<0.001$, see Fig.7a red nodes). The disease module apparently contains many genes that are not significantly associated with the disease (i.e., with GWAS gene-wise p-value $>=0.001$, see Fig.7a blue nodes). Those genes are "absorbed" into the disease module due to their direct connection with those genes with very low GWAS p-values. We further performed the functional enrichment analysis of genes in the disease module using gprofiler $2^{40}$. We found that these genes in the disease module are associated with many functional terms (see Fig.7b). Finally, we showed the top-10 enriched KEGG pathways (with lowest p-values in Fig.7b) enriched with genes in the 
230 disease module (see Fig.7c). We found that these pathways are potentially quite relevant to asthma.

231 For example, proteasome inhibition is a novel mechanism to combat asthma ${ }^{41}$; Spliceosome are 232 involved with the development of severe asthma ${ }^{42}$ and Notch signaling links embryonic lung 233 development and asthmatic airway remodeling ${ }^{43}$. See Fig.S3 and S4 for the disease modules and 234 functional terns of other disease phenotypes.

\section{Literature validation for SNPs/genes in disease modules identified from GWAS}

237 Finally, we explored the biological relevance of these genes belonging to the disease module. To narrow down the validation space, we focused on the common genes simultaneously identified by all three methods: RFIM, DIAMOnD and ModuleDiscoverer. We anticipate that those common genes are more important than genes identified by a single method. Interestingly, we found that those common genes were typically not significantly associated with the disease (based on the GWAS gene-wise $p$-values $>=0.001$ (see Table 2). Yet, almost all these genes have been shown expression and activity are downregulated and inversely correlated with lung function ${ }^{44}$. For asthma, the gene IL9 has been implicated as an essential factor in determining susceptibility to atopic asthma and a therapeutic target for asthma ${ }^{45}$. For lung cancer, CD74 has been associated with many tumor cells, i.e., non-small and small cell lung cancer ${ }^{46,47}$. And as a component of the HLA class-I complex, B2M has been shown to have recurrent inactivation in lung cancer. This was proposed to be an acquired mechanism for avoiding tumor immune recognition. ${ }^{48}$ For CVD, $A P O C 3$, an important regulator of TG homeostasis, is beneficially associated with decreased CVD risk $^{49-51}$. These results imply that the genes in the disease module can be potentially associated with specific diseases despite their non-significance based on the current GWAS.

\section{DISCUSSION}

A complex disease can be linked to a well-defined neighborhood of the cellular molecular interactome, referred to as the disease module. Detecting the disease module allows us to identify driver genes that are heavily involved in the disease. Those driver genes do not necessarily have significant p-values in GWAS or other omics data, and hence cannot be easily identified without leveraging the human interactome. Here, we developed a novel disease module detection method based on a statistical physics model RFIM, which is both mathematically rigorous and computationally efficient. We applied our method to analyze six GWAS datasets, covering welldefined complex disease phenotypes. We found that the disease modules identified by RFIM display higher connectivity and more enriched pathways than the other existing methods.

264 Furthermore, we found literature evidence that those genes in the disease module (especially those 265 common genes identified by different methods) have significant associations with the specific 266 disease.

Our RFIM approach can be naturally used to integrate other omics data, e.g., gene expression and DNA methylation, with the interactome. We admit that applying a disease module detection method via integrating omics data and the human interactome sometimes requires an 
annotation, which might lead to inaccuracy. For example, GWAS signals ${ }^{32,52-54}$ do not provide precise localization to a gene and the affected gene could be located at great genomic distance. Yet, we expect that using nearby genes is accurate enough to create meaningful disease modules.

\section{METHODs}

\section{Calculation of the $\boldsymbol{M}(\boldsymbol{H})$ curve of RFIM.}

276 To calculate the $M(H)$ curve of RFIM, we first need to calculate the exact ground state of the system at an arbitrary applied external field $H$. This is the basic step of calculating the $M(H)$ curve, i.e., the ground state evolution for varying $H$. Fortunately, there is a well-known mapping of the ground state problem of RFIM to a min-cut/max-flow problem in combinatorial optimization. The mapping and the so-called push-relabel algorithm for the min-cut/max-flow problem has been well described in the literature ${ }^{22}$. The run time of the push-relabel algorithm ${ }^{22}$ scales as $O\left(N^{4 / 3}\right)$ with $N$ the system size (number of nodes). The $M(H)$ curve can be calculated with the method reported in $\left[{ }^{49,50}\right]$. It is essentially based on the fact that the ground state energy is convex up in $H$, which allows for estimates of the fields $H$ where the magnetization jumps (called "avalanches") occur. This algorithm finds steps by narrowing down ranges where the magnetization jumps with an efficient linear interpolation scheme ${ }^{55}$. Applying the no-passing rule (i.e., flipped spins can never flip back with increasing $H$ ) can further speed up the calculation of the RFIM $M(H)$ curve $^{31}$.

\section{Datasets}

290 Interactome. For the human interactome, we used the STRING ${ }^{33}$ (9606.protein.links.v11.5 .txt) database (http://string-db.org), which aims to provide a critical assessment and integration of PPIs, including both direct (physical) and indirect (functional) associations. We removed the PPIs whose normalized scores are lower than 0.7. The STRING Ensemble protein id (ENSP) is transformed into preferred gene name (e.g., entrez gene ID) using the protein information (9606.protein.info.v11.5.txt) downloaded from the STRING database. To examine the impact of different interactomes on the disease module detection methods, we also employed another interactome iRefIndex ${ }^{34,35}$ from the dataset extracted using $\mathrm{R}$ package cisPath $^{57}$ (version 1.28.0).

GWAS. We used MAGMA ${ }^{29}$ (Multi-marker Analysis of GenoMic Annotation) to analyze the GWAS data. In MAGMA, SNPs were annotated to the genes based on dbSNP version 135 SNP location and NCBI 37.3. Two gene annotations were used in MAGMA. Only SNPs located between a gene's transcription start and stop sites were annotated to that gene for the main analyses and a 10 kilobase window around each gene was made in additional annotation ${ }^{29}$. The GWAS data of CVD was downloaded from http://www.cardiogramplusc4d.org/data-downloads/ (version 2015). The GWAS data of diabetes was downloaded from http://diagramconsortium.org/downloads.html (version 2016). The GWAS data of COPD was collected in Ref [24], which combines 26 cohorts containing 63,192 individuals (15,256 COPD cases and 47,936 controls). The p-values and annotations of SNPs for CVD, diabetes and COPD were obtained using MAGMA. For the asthma, breast cancer and lung cancer data sets, the p-values of SNPs were 
310 directly downloaded from http://jenger.riken.jp/en/result and SNPs have been annotated to the 311 genes in the summary results. The $\mathrm{p}$-value of each gene in all datasets was obtained using mean $312 \chi^{2}$ statistic of SNPs included in the gene and a known approximation of the sampling distribution 313 of the mean $\chi^{2}$ statistic $^{27,28}$ using MAGMA.

\section{Existing disease module detection method used for comparison}

316 There have many existing phenotype-specific disease module detection methods. For example, the 317 DIseAse Module Detection (DIAMOnD) algorithm ${ }^{18}$ starts from a set of seed genes and prioritizes 318 the other proteins of the interactome for their putative disease relevance. Therefore, the identified disease module might significantly rely on the initial seed genes. Another method, DysrEgulated Gene set Analysis via Subnetworks (DEGAS) detects the subnetworks in which multiple genes are dysregulated in the cases ${ }^{15}$. In the first step, the set of genes that are dysregulated when compared to controls are identified. Then, DEGAS identifies the smallest subgraph that contains at least $k$ genes from each of those sets, except for up to $l$ outlier sets from which fewer genes can be present. Parameters $k$ and $l$ are used to control the number of genes affected in the pathways in each individual and the number of allowed outliers, respectively, and several heuristics and algorithms were proposed to find these minimally connected subnetworks. In this work, we compared our proposed RFIM method with existing disease module detection methods incorporated in R package MODifieR ${ }^{14}$ which can simultaneously use the PPI network and genewise p-values. (1) ModuleDiscoverer ${ }^{17}$. ModuleDiscoverer uses a randomization heuristic-based approximation of the community structure based on maximal clique enumeration problem. (2) DIAMOnD $^{18}$. DIAMOnD starts from a set of seed genes and prioritizes the other proteins of the interactome for their putative disease relevance. These methods are originally designed for gene expression data. The number of genes in the disease module in DIAMOnD set to be 500 . We used the gene-wise p-value from GWAS as the input to ModuleDiscoverer and DIAMOnD. 
Table 1 Summary of GWAS studies used in the disease module detection.

\begin{tabular}{l|llll}
\hline Phenotype & \# Genes & \# Cases & \# Controls & Ref \\
\hline COPD & 17,866 & 15,256 & 47,936 & 24 \\
CVD & 17,866 & 60,801 & 123,504 & 25 \\
Diabetes & 17,894 & 11,645 & 32,769 & 26 \\
Asthma & 17,260 & 8,216 & 201,592 & 23 \\
Breast cancer & 17,215 & 5,552 & 89,731 & 23 \\
Lung cancer & 17,261 & 4,050 & 208,403 & 23 \\
\hline
\end{tabular}

351

352

353

354

355

356

357

358

359

360

361 
a
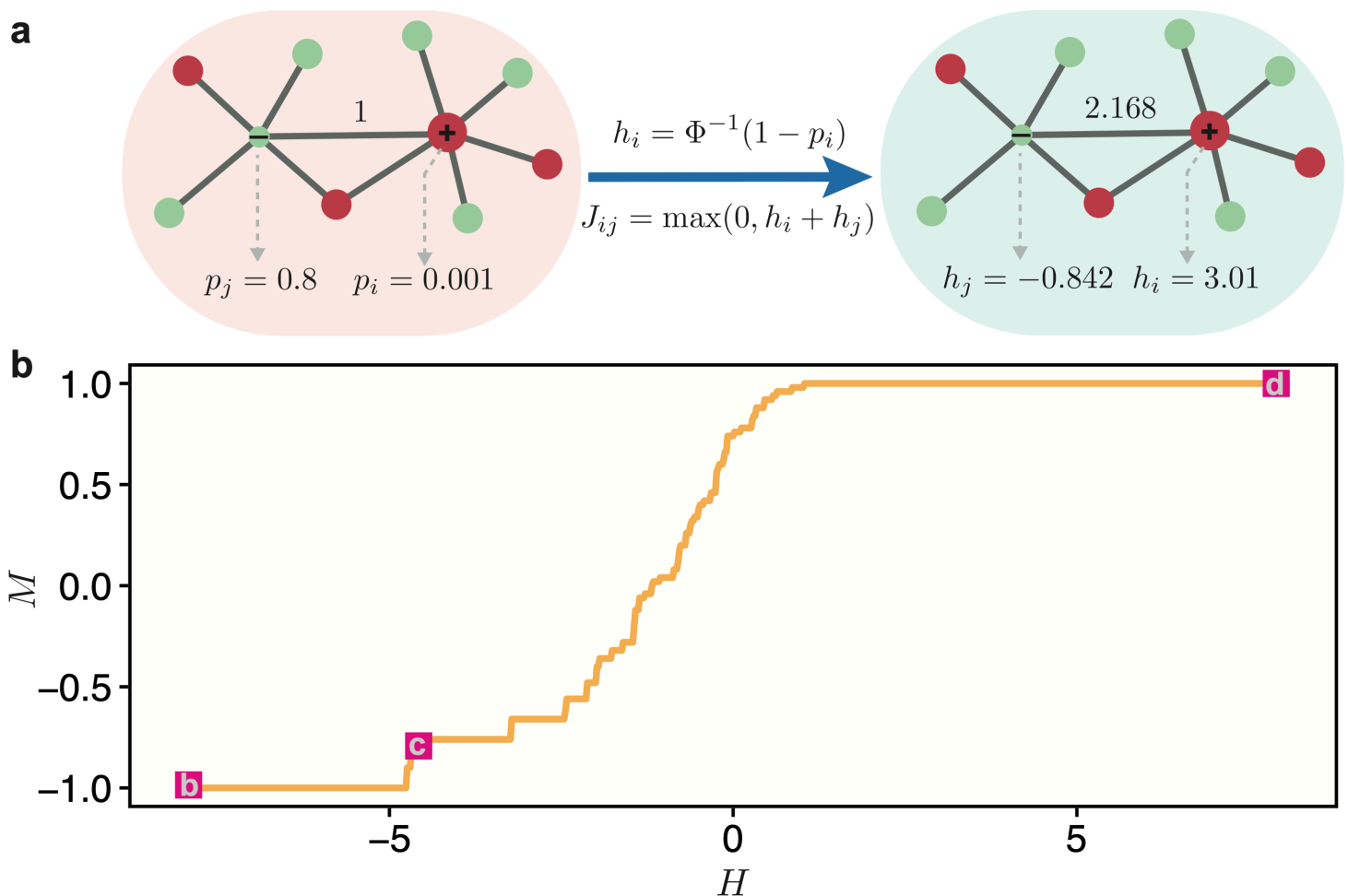

C

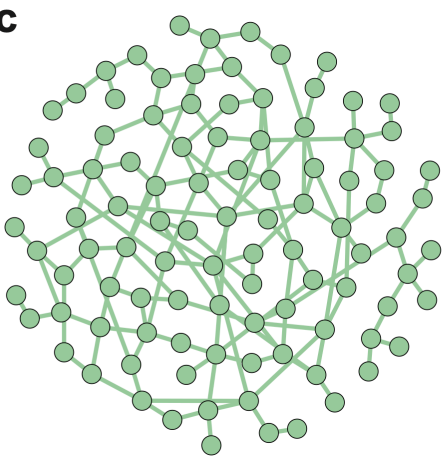

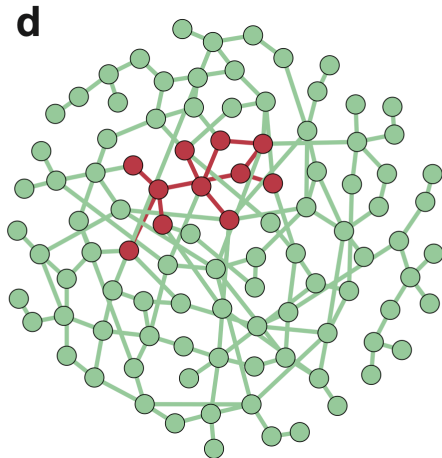

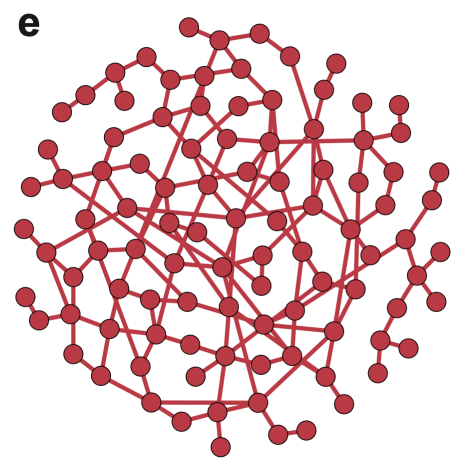

Figure 1: Disease module inference using statistical physics approach: a demonstration. (a): Schematic demonstration of the RFIM approach. A random network with $N=100$ nodes (genes). Each gene is assigned a p-value, randomly chosen from a uniform distribution $U(0,1)$. The node weights $h_{i}$ are then computed using the inverse normal CDF. The edge weights $J_{i j}=\max \left(0, h_{i}+\right.$ $h_{j}$ ) if two genes are connected in the network. Otherwise, $J_{i j}=0$. (b): The magnetization curve $M(H)$ of the random network system. The magnetization $M \equiv 1 / N \sum_{i=1}^{N} \sigma_{i}$ is the average state of nodes in the system. As we increase the external field $H$, more nodes (genes) will be active, i.e., $M$ will monotonically increase and eventually approach 1 . (c-e): disease module identified by RFIM at $H=-8$ (point b), $H=-4$ (point c) and $H=8$ (point d), respectively. All the active components (highlighted in red) together can be considered as the disease module. 
a STRING
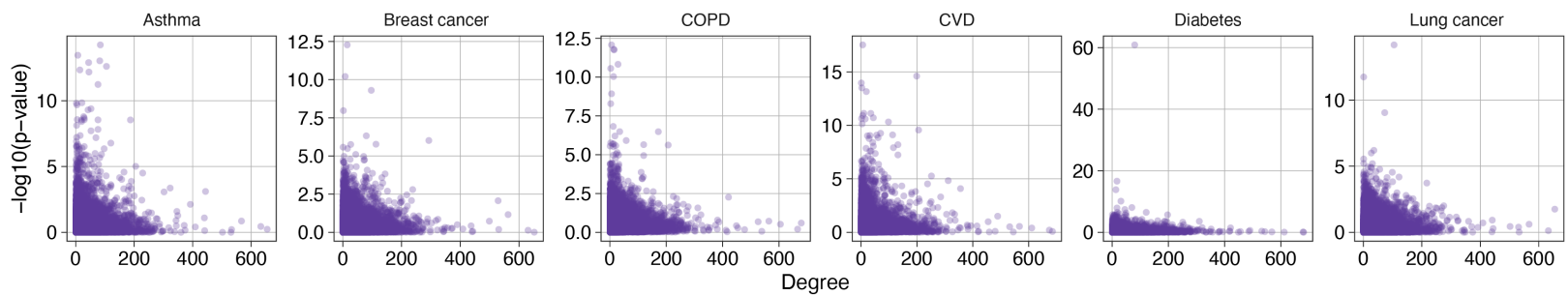

b iReflndex
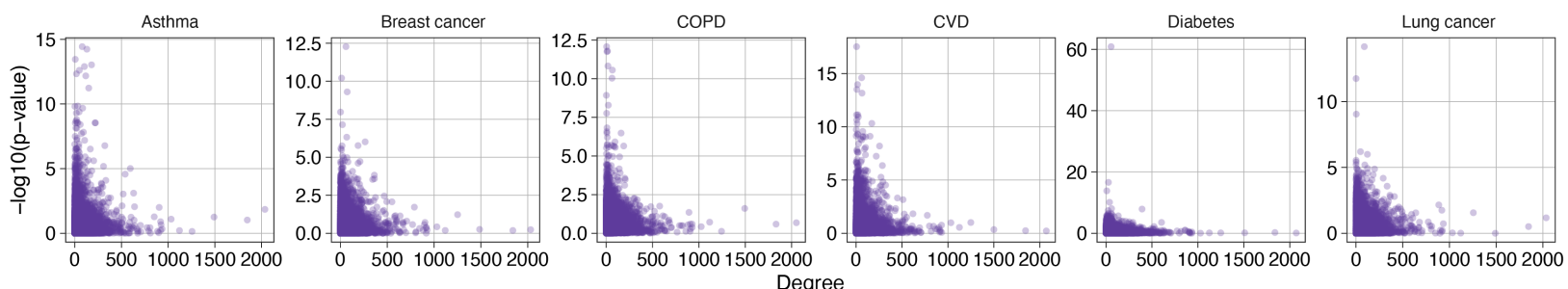

Figure 2: Association between gene-wise p-value and gene degree. For each phenotype, we showed the gene-wise p-value $(\log 10$ scale) vs. its degree in the interactome of (a): STRING and (b): iRefIndex. 


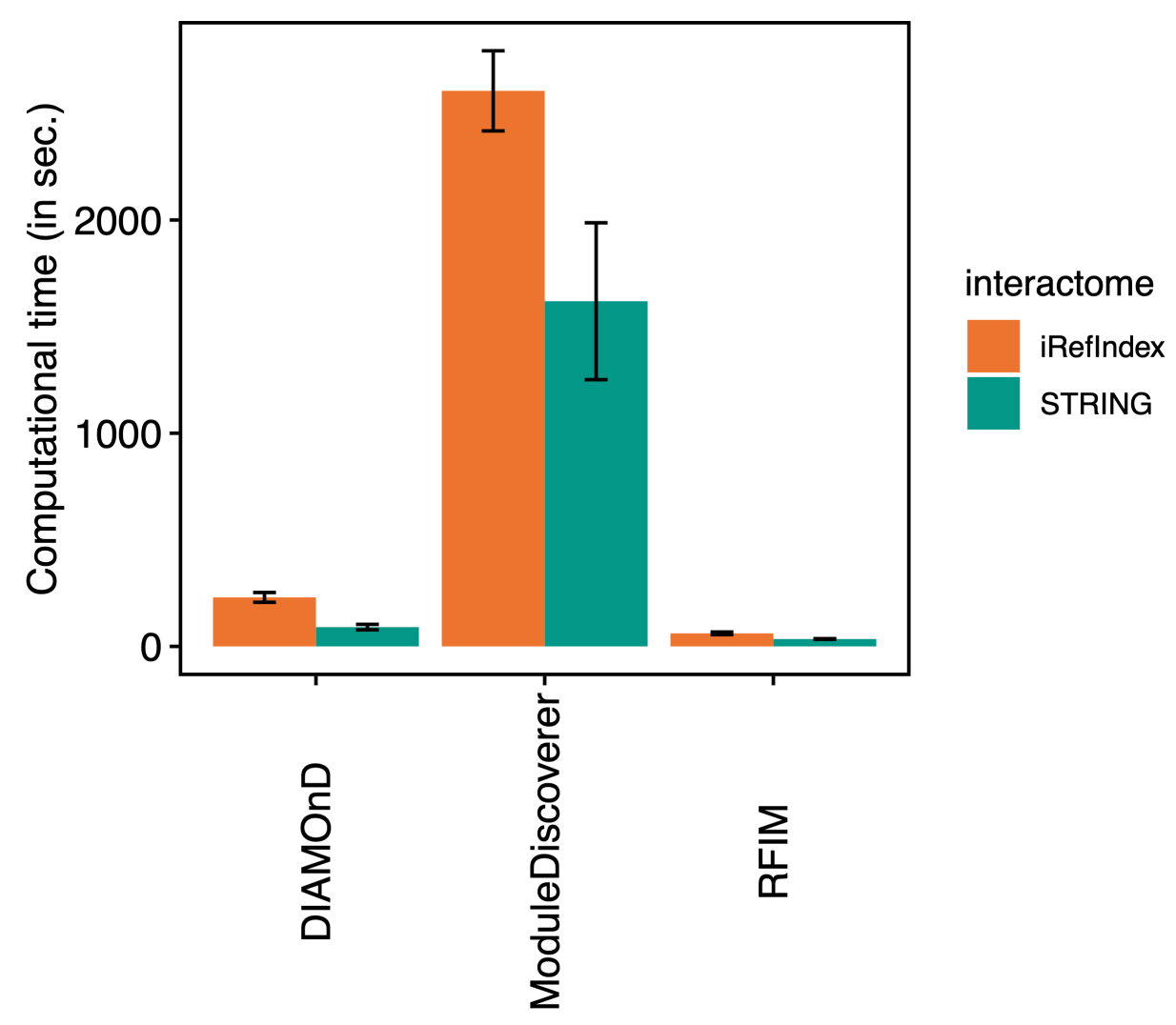

386 Figure 3: Computational complexity of each disease module detection method. Each bar 387 represents the average computational time (in seconds) overall all six phenotypes. Error bar 388 represents the standard derivation among 6 GWAS datasets. The computational time is measured 389 as the running time on macOS machine with $2.4 \mathrm{GHz} 8$-Core Intel Core i9 processor. 
403

\section{a STRING}
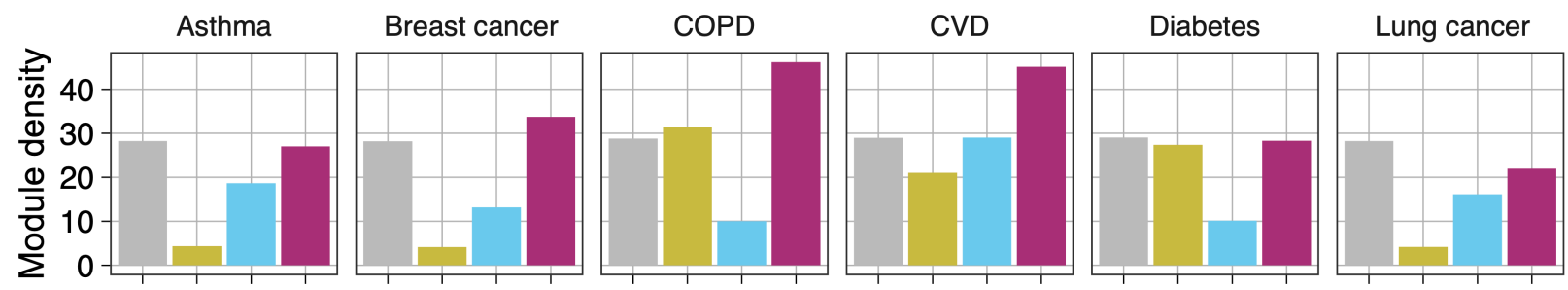

\section{b iReflndex}
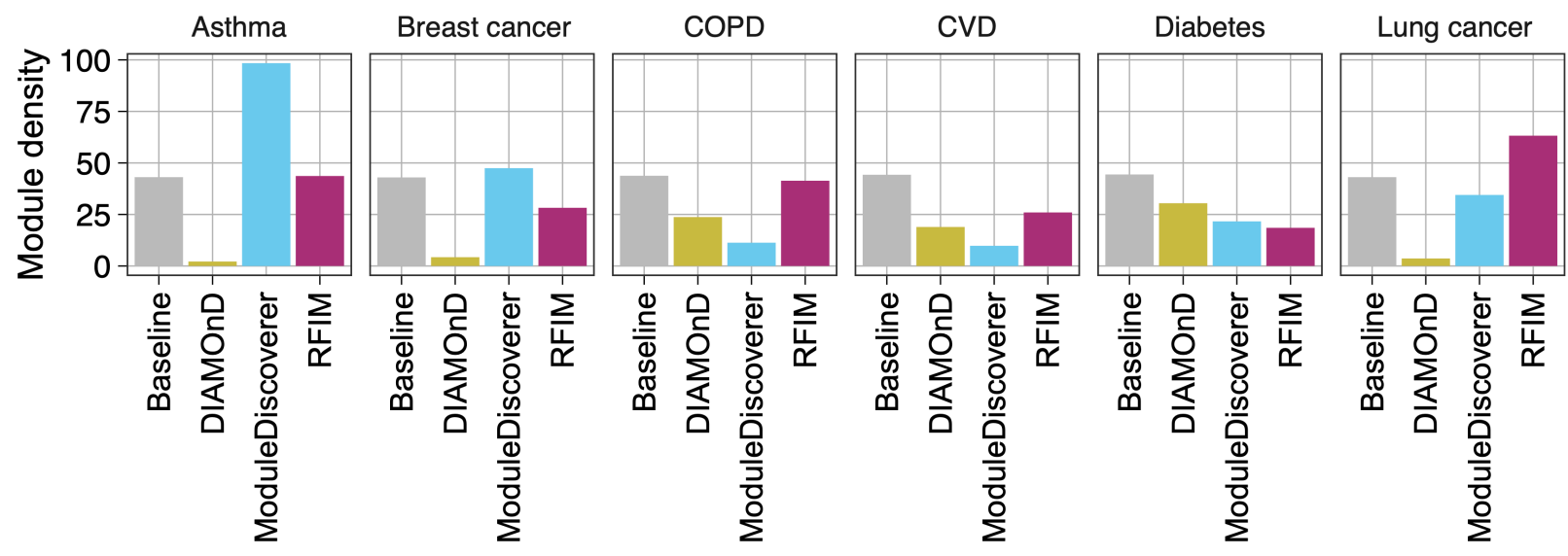

Figure 4: Mean degree of disease module for each phenotype and method. We applied each disease module detection method in the GWAS of each disease separately. Baseline represents the average degree of the interactome. For each disease, we chose to work on the common genes between the interactome and the GWAS, thus the average degree of the interactome in different diseases slightly varies.

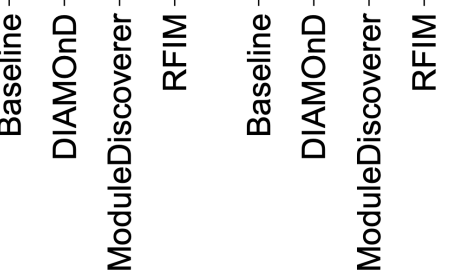


a STRING
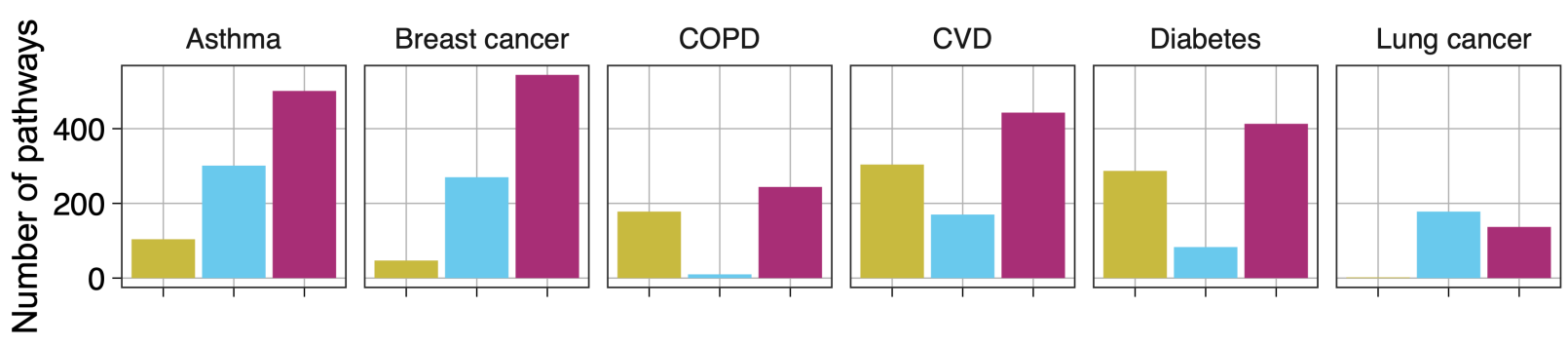

\section{b iReflndex}
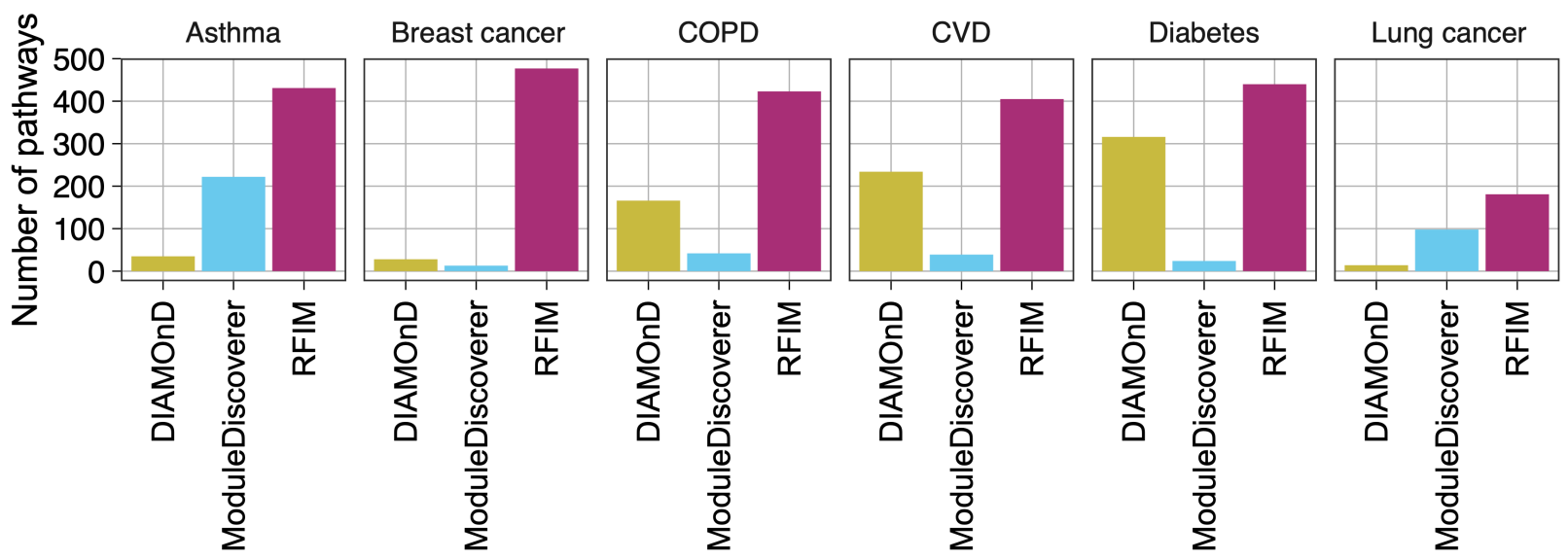

Figure 5: Enrichment analysis for genes in disease module detected from integrating the GWAS with two interactomes for each phenotype and method. We obtained the enriched pathways for disease genes using the ReactomePA package ${ }^{38}$ whose p-values are lower than 0.05 cutoff adjust by False Discovery Rate (FDR). Then, we extracted the disease-associated genes of each phenotype using the DisGeNET ${ }^{39}$ database and calculated the number of enriched pathways with at least two disease-associated genes. 

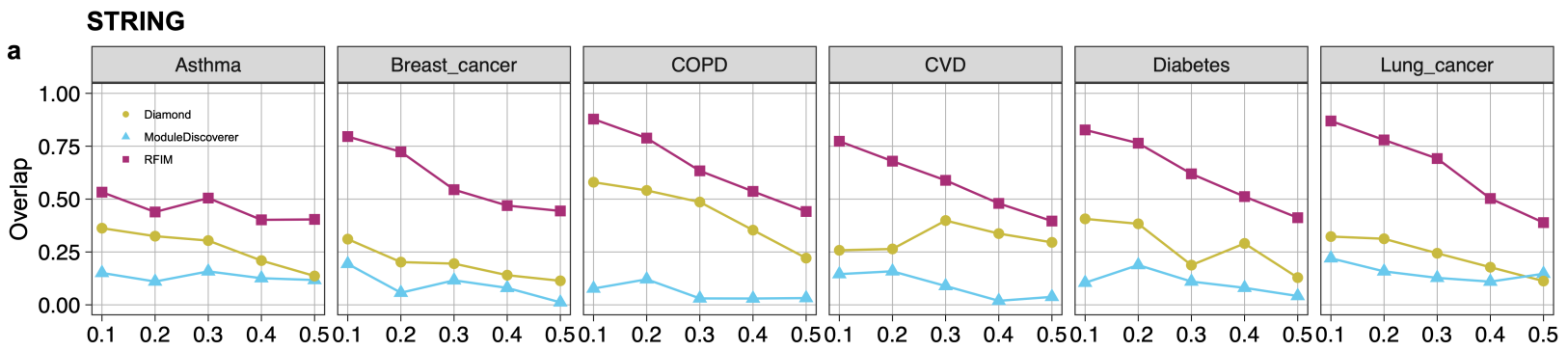

b iRefIndex
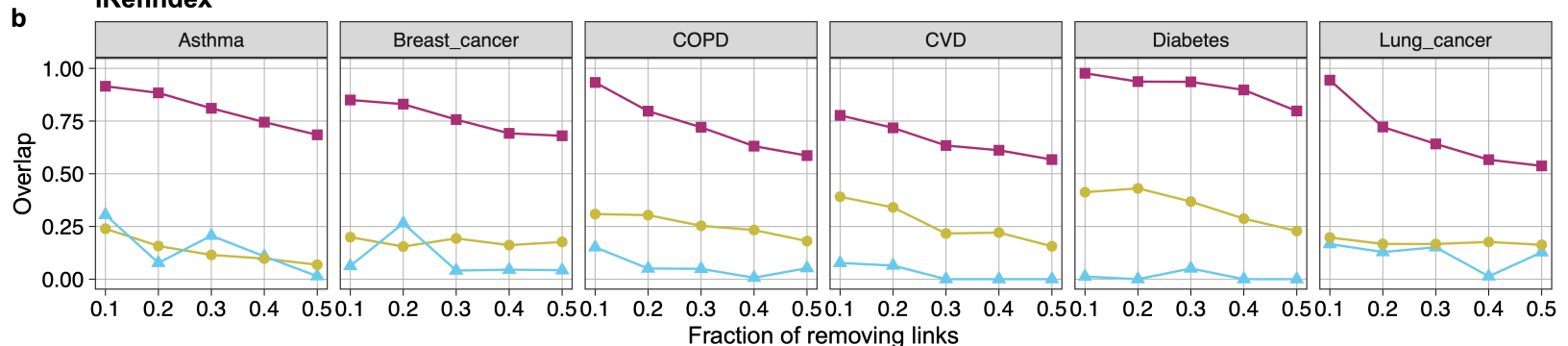

Figure 6: Sensitivity of disease module detection to the interactome. For each interactome, STRING (a) or iRefIndex (b), we firstly applied each disease module into the original interactome. Then, a fraction of PPIs randomly selected from the original interactome were removed. Finally, we performed the disease module detection algorithms again using perturbed interactome and compared the fraction of overlapping genes in the union genes of the disease module identified 
a

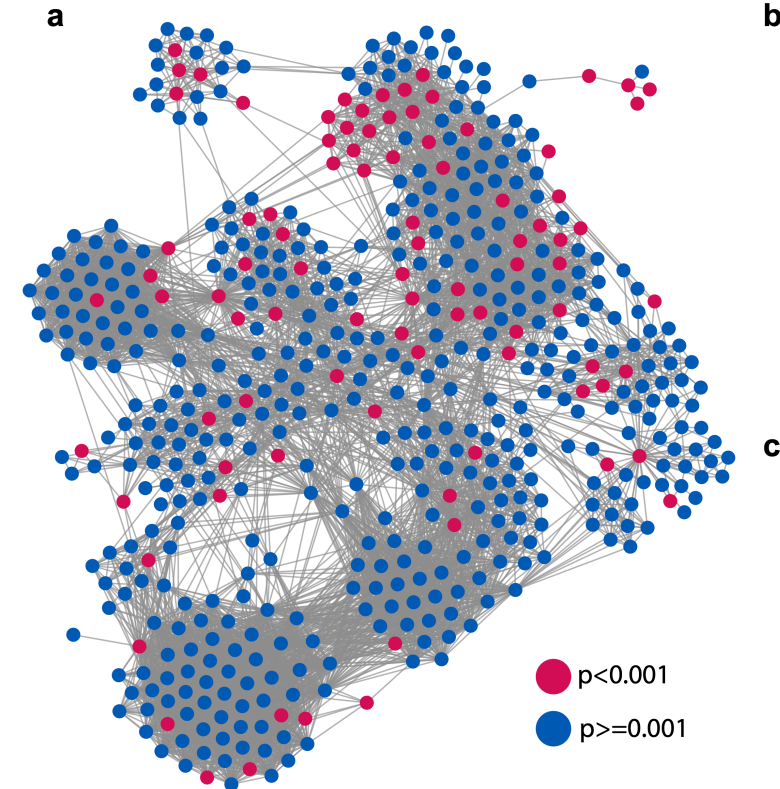

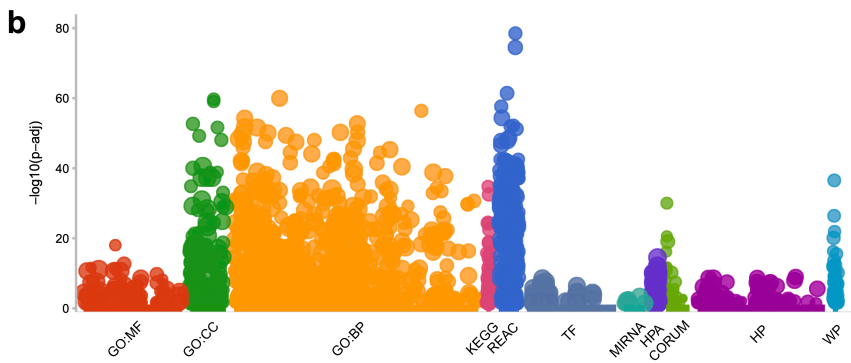

\begin{tabular}{|l|l|l|l|l|l|l|}
\hline id & source & term_id & \multicolumn{1}{|c|}{ term_name } & term_size & intersection_size & P_value \\
\hline 1 & KEGG & KEGG:03050 & Proteasome & 46 & 36 & $1.6 e-35$ \\
\hline 2 & KEGG & KEGG:04658 & Th1 and Th2 cell differentiation & 89 & 46 & $3.2 e-33$ \\
\hline 3 & KEGG & KEGG:03040 & Spliceosome & 150 & 50 & $4.5 e-25$ \\
\hline 4 & KEGG & KEGG:04330 & Notch signaling pathway & 59 & 33 & $5.5 e-25$ \\
\hline 5 & KEGG & KEGG:05169 & Epstein-Barr virus infection & 198 & 55 & $3.0 \mathrm{e}-23$ \\
\hline 6 & KEGG & KEGG:04612 & Antigen processing and presentation & 69 & 31 & $1.2 \mathrm{e}-19$ \\
\hline 7 & KEGG & KEGG:04659 & Th17 cell differentiation & 104 & 37 & $2.3 \mathrm{e}-19$ \\
\hline 8 & KEGG & KEGG:05330 & Allograft rejection & 34 & 21 & $8.2 \mathrm{e}-17$ \\
\hline 9 & KEGG & KEGG:00052 & Galactose metabolism & 31 & 20 & $1.6 \mathrm{e}-16$ \\
\hline 10 & KEGG & KEGG:05017 & Spinocerebellar ataxia & 142 & 39 & $6.3 \mathrm{e}-16$ \\
\hline
\end{tabular}

Figure 7: Sub-modules in the asthma disease module and the enriched functional terms. (a):

Genes in the asthma disease module form several sub-modules. Genes with p-values lower (or higher) than 0.001 are colored with red (or blue), respectively. (b): Enriched functional terms of genes in disease module of asthma using gprofier $2^{40}$. (c): Top-10 enriched KEGG pathways of genes in the asthma disease module using STRING. 
Table 2 Biological relevance of genes simultaneously identified by three disease module detection methods

\begin{tabular}{|c|c|c|c|}
\hline Phenotype & Gene & Association with disease & Ref \\
\hline Asthma & IL9 & $\begin{array}{l}\text { IL9 has been implicated as an essential factor in determining susceptibility to atopic asthma and } \\
\text { a therapeutic target for asthma. }\end{array}$ & 58 \\
\hline $\begin{array}{l}\text { Breast } \\
\text { cancer }\end{array}$ & BANF1 & $\begin{array}{l}\text { Expression of BANF1 may play a role on the occurrence of development of triple-negative } \\
\text { breast cancer. }\end{array}$ & 59 \\
\hline \multirow[t]{10}{*}{ COPD } & $\begin{array}{l}\text { PSMC6 } \\
\text { PSMB1 } \\
\text { PSMD12 }\end{array}$ & $\begin{array}{l}\text { Downregulated different expressed gene in COPD identified by three microarray datasets } \\
\text { integrated using the Robust Rank Aggregation (RRA) method. } \\
\text { PSMB1 is lower in the COPD group than in the control group. } \\
\text { * }\end{array}$ & 61 \\
\hline & PSMD8 & $\begin{array}{l}\text { PSMD8 was reported to be more highly expressed in sedentary COPD patients than in sedentary } \\
\text { controls. }\end{array}$ & 62 \\
\hline & PSMD11 & Differentially expressed in malignant bronchial epithelial cell line compared to control cell line. & 63 \\
\hline & PSMB4 & $\begin{array}{l}\text { PSMB4 reveals significant upregulation, different expression and association with proliferation } \\
\text { in human pulmonary neuroendocrine tumors. }\end{array}$ & 64 \\
\hline & PSMC3 & PSMC3 is down-regulated gene in COPD. & 65 \\
\hline & PSMD3 & PSMD3 has been reported to associate to atopic march and to atopic eczema and wheeze. & 66 \\
\hline & PSMC4 & PSMC4 is down-regulated gene in COPD. & 65 \\
\hline & PSMD6 & $\begin{array}{l}\text { A downregulated gene between six- and eightfold in peripheral blood mononuclear cells of } \\
\text { patients with stable COPD or AECOPD compared to healthy control subjects. }\end{array}$ & 67 \\
\hline & PSMD13 & PSMD13 is down-regulated gene in COPD. & 61 \\
\hline & KIAA2012 & Upregulated differentially expressed gene in COPD. & 60 \\
\hline \multirow[t]{14}{*}{ CVD } & APOC4 & $\begin{array}{l}\text { APOC4 (rs1132899 polymorphism) was associated with an increased risk of premature CAD } \\
\text { in Chinese subjects. }\end{array}$ & 68 \\
\hline & APOC2 & The CHD patients had a higher mean concentration of APOC2 than controls. & 69 \\
\hline & APOC3 & $\begin{array}{l}\text { APOC3, an important regulator of TG homeostasis, is beneficially associated with decreased } \\
\text { CVD risk. }\end{array}$ & $49-51$ \\
\hline & $\mathrm{APOH}$ & $\mathrm{APOH}$ is increased in the plasma and liver of type 2 diabetic patients with metabolic syndrome. & 70 \\
\hline & APOA4 & $\begin{array}{l}\text { Two polymorphisms were associated with increased CVD risk, namely the Met } 235 \mathrm{Thr} \text { variant } \\
\text { in the angiotensinogen gene and the Thr } 347 \text { Ser variant in the apoA4 gene. }\end{array}$ & 71 \\
\hline & APOL1 & $A P O L 1$ risk variants display a modest association with CVD. & 72 \\
\hline & PCYOX1 & PCYOX1 a potential pharmacological target and a new biomarker in cardiovascular disease. & 73 \\
\hline & PLTP & Higher PLTP activity was each associated with a significantly increased risk of CVD. & 74 \\
\hline & CLU & $\begin{array}{l}\text { Clusterin is significantly related to the most atherogenic components of lipid profile, especially } \\
\text { in women, suggesting its potential role in modulating cardiovascular metabolic risk factors. }\end{array}$ & 75 \\
\hline & PLA2G7 & PLA2G7 is a cardiovascular risk marker independent of and additive to traditional risk factors. & 76 \\
\hline & DBI & * & \\
\hline & PEX3 & * & \\
\hline & LCAT & $\begin{array}{l}\text { LCAT protects against atherosclerosis. This in turn indicates that targeting LCAT to raise HDL- } \\
\text { C may reduce CVD risk. }\end{array}$ & 77 \\
\hline & ACTA1 & $\begin{array}{l}\text { ACTA1, NPPB and MYH7 are known to be induced in stressed cardiomyocytes in infarcted, } \\
\text { hypertrophic ad dilated hearts and some of these genes have been used for diagnosis and } \\
\text { prognosis in heart disease. }\end{array}$ & 78 \\
\hline \multirow[t]{2}{*}{$\begin{array}{l}\text { Lung } \\
\text { cancer }\end{array}$} & CD74 & $\begin{array}{l}\text { CD74 has been shown association with many tumor cells, i.e., non-small and small cell lung } \\
\text { cancer. }\end{array}$ & 46,47 \\
\hline & $\mathrm{B} 2 \mathrm{M}$ & $\begin{array}{l}\text { As a component of the HLA class-I complex, B2M has been shown to have recurrent } \\
\text { inactivation in lung cancer. This was proposed to be an acquired mechanism for avoiding } \\
\text { tumor immune recognition. }\end{array}$ & 48 \\
\hline
\end{tabular}




\section{References}

1. Cho, D.-Y., Kim, Y.-A. \& Przytycka, T. M. Chapter 5: Network biology approach to complex diseases. PLoS Comput. Biol. 8, e1002820 (2012).

2. Barabási, A.-L., Gulbahce, N. \& Loscalzo, J. Network medicine: a network-based approach to human disease. Nat. Rev. Genet. 12, 56-68 (2011).

3. Gustafsson, M. et al. Modules, networks and systems medicine for understanding disease and aiding diagnosis. Genome Med. 6, 82 (2014).

4. Zanzoni, A., Soler-López, M. \& Aloy, P. A network medicine approach to human disease. FEBS Lett. 583, 1759-1765 (2009).

5. Goh, K.-I. et al. The human disease network. Proc. Natl. Acad. Sci. 104, 8685-8690 (2007).

6. Oti, M., Snel, B., Huynen, M. A. \& Brunner, H. G. Predicting disease genes using proteinprotein interactions. J. Med. Genet. 43, 691-698 (2006).

7. $\mathrm{Xu}, \mathrm{J} . \& \mathrm{Li}, \mathrm{Y}$. Discovering disease-genes by topological features in human protein-protein interaction network. Bioinformatics 22, 2800-2805 (2006).

8. Gandhi, T. K. B. et al. Analysis of the human protein interactome and comparison with yeast, worm and fly interaction datasets. Nat. Genet. 38, 285-293 (2006).

9. Chu, L.-H. \& Chen, B.-S. Construction of a cancer-perturbed protein-protein interaction network for discovery of apoptosis drug targets. BMC Syst. Biol. 2, 1-17 (2008).

10. Azmi, A. S., Wang, Z., Philip, P. A., Mohammad, R. M. \& Sarkar, F. H. Proof of concept: network and systems biology approaches aid in the discovery of potent anticancer drug combinations. Mol. Cancer Ther. 9, 3137-3144 (2010).

11. Zhao, S. \& Li, S. Network-based relating pharmacological and genomic spaces for drug target identification. PloS One 5, e11764 (2010).

12. Cheng, F., Kovács, I. A. \& Barabási, A.-L. Network-based prediction of drug combinations. Nat. Commun. 10, 1197 (2019).

13. Morselli Gysi, D. et al. Network medicine framework for identifying drug-repurposing opportunities for COVID-19. Proc. Natl. Acad. Sci. 118, e2025581118 (2021).

14. de Weerd, H. A. et al. MODifieR: an Ensemble R Package for Inference of Disease Modules from Transcriptomics Networks. Bioinformatics 36, 3918-3919 (2020).

15. Ulitsky, I., Krishnamurthy, A., Karp, R. M. \& Shamir, R. DEGAS: De Novo Discovery of Dysregulated Pathways in Human Diseases. PLoS ONE 5, e13367 (2010).

16. Bersanelli, M., Mosca, E., Remondini, D., Castellani, G. \& Milanesi, L. Network diffusionbased analysis of high-throughput data for the detection of differentially enriched modules. Sci. Rep. 6, 34841 (2016).

17. Vlaic, S. et al. ModuleDiscoverer: Identification of regulatory modules in protein-protein interaction networks. Sci. Rep. 8, 433 (2018).

18. Ghiassian, S. D., Menche, J. \& Barabási, A.-L. A DIseAse MOdule Detection (DIAMOnD) Algorithm Derived from a Systematic Analysis of Connectivity Patterns of Disease Proteins in the Human Interactome. PLOS Comput. Biol. 11, e1004120 (2015).

19. Belanger, D. P. \& Young, A. P. The random field Ising model. J. Magn. Magn. Mater. 100, 272-291 (1991).

20. Bray, A. J. \& Moore, M. A. Scaling theory of the random-field Ising model. J. Phys. C Solid State Phys. 18, L927 (1985).

21. d'Auriac, J. A., Preissmann, M. \& Rammal, R. The random field Ising model: algorithmic complexity and phase transition. J. Phys. Lett. 46, 173-180 (1985). 
22. Cherkassky, B. V. \& Goldberg, A. V. On implementing the push-relabel method for the maximum flow problem. Algorithmica 19, 390-410 (1997).

23. Ishigaki, K. et al. Large-scale genome-wide association study in a Japanese population identifies novel susceptibility loci across different diseases. Nat. Genet. 52, 669-679 (2020).

24. Hobbs, B. D. et al. Genetic loci associated with chronic obstructive pulmonary disease overlap with loci for lung function and pulmonary fibrosis. Nat. Genet. 49, 426-432 (2017).

25. Schunkert, H. et al. Large-scale association analysis identifies 13 new susceptibility loci for coronary artery disease. Nat. Genet. 43, 333-338 (2011).

26. Mehta, N. N. Large-scale association analysis provides insights into the genetic architecture and pathophysiology of type 2 diabetes mellitus. Circ. Cardiovasc. Genet. 5, 708-710 (2012).

27. Brown, M. B. 400: A method for combining non-independent, one-sided tests of significance. Biometrics 987-992 (1975).

28. Hou, C.-D. A simple approximation for the distribution of the weighted combination of nonindependent or independent probabilities. Stat. Probab. Lett. 73, 179-187 (2005).

29. de Leeuw, C. A., Mooij, J. M., Heskes, T. \& Posthuma, D. MAGMA: Generalized Gene-Set Analysis of GWAS Data. PLOS Comput. Biol. 11, e1004219 (2015).

30. NATTERMANN, T. Theory of the random field Ising model. in Spin glasses and random fields 277-298 (World Scientific, 1998).

31. Liu, Y. \& Dahmen, K. A. No-passing rule in the ground state evolution of the random-field Ising model. Phys. Rev. E 76, 031106 (2007).

32. Regan, E. A. et al. Genetic Epidemiology of COPD (COPDGene) Study Design. COPD 7, 32-43 (2010).

33. Franceschini, A. et al. STRING v9. 1: protein-protein interaction networks, with increased coverage and integration. Nucleic Acids Res. 41, D808-D815 (2012).

34. Razick, S., Magklaras, G. \& Donaldson, I. M. iRefIndex: a consolidated protein interaction database with provenance. BMC Bioinformatics 9, 1-19 (2008).

35. Aranda, B. et al. PSICQUIC and PSISCORE: accessing and scoring molecular interactions. Nat. Methods 8, 528-529 (2011).

36. Zotenko, E., Mestre, J., O'Leary, D. P. \& Przytycka, T. M. Why Do Hubs in the Yeast Protein Interaction Network Tend To Be Essential: Reexamining the Connection between the Network Topology and Essentiality. PLoS Comput. Biol. 4, e1000140 (2008).

37. Wang, T. et al. Identification and characterization of essential genes in the human genome. Science 350, 1096-1101 (2015).

38. Yu, G. \& He, Q.-Y. ReactomePA: an R/Bioconductor package for reactome pathway analysis and visualization. Mol. Biosyst. 12, 477-479 (2016).

39. Piñero, J. et al. DisGeNET: a comprehensive platform integrating information on human disease-associated genes and variants. Nucleic Acids Res. gkw943 (2016).

40. Kolberg, L., Raudvere, U., Kuzmin, I., Vilo, J. \& Peterson, H. gprofiler2 -- an R package for gene list functional enrichment analysis and namespace conversion toolset g:Profiler. F1000Research 9, ELIXIR-709 (2020).

41. Elliott, P. J., Pien, C. S., McCormack, T. A., Chapman, I. D. \& Adams, J. Proteasome inhibition: A novel mechanism to combat asthma㑔㶦. J. Allergy Clin. Immunol. 104, 294 300 (1999). 
601

602

603

604

605

606

607

608

609

610

611

612

613

614

615

616

617

618

619

620

621

622

623

624

625

626

627

628

42. Comprehensive analysis of miRNA-mRNA-lncRNA networks in severe asthma. https://www.futuremedicine.com/doi/epub/10.2217/epi-2018-0132 doi:10.2217/epi-20180132.

43. Hussain, M. et al. Notch Signaling: Linking Embryonic Lung Development and Asthmatic Airway Remodeling. Mol. Pharmacol. 92, 676-693 (2017).

44. Meiners, S. \& Eickelberg, O. What shall we do with the damaged proteins in lung disease? Ask the proteasome! Eur. Respir. J. 40, 1260-1268 (2012).

45. Zhou, Y., McLane, M. \& Levitt, R. C. Th2 cytokines and asthma - Interleukin-9 as a therapeutic target for asthma. Respir. Res. 2, 80-84 (2001).

46. Gou, W. et al. CD74-ROS1 G2032R mutation transcriptionally up-regulates Twist1 in nonsmall cell lung cancer cells leading to increased migration, invasion, and resistance to crizotinib. Cancer Lett. 422, 19-28 (2018).

47. Song, Y., Sun, Y., Lei, Y., Yang, K. \& Tang, R. YAP1 promotes multidrug resistance of small cell lung cancer by CD74-related signaling pathways. Cancer Med. 9, 259-268 (2020).

48. Pereira, C. et al. Genomic Profiling of Patient-Derived Xenografts for Lung Cancer Identifies B2M Inactivation Impairing Immunorecognition. Clin. Cancer Res. 23, 3203-3213 (2017).

49. Dib, I., Khalil, A., Chouaib, R., El-Makhour, Y. \& Noureddine, H. Apolipoprotein C-III and cardiovascular diseases: when genetics meet molecular pathologies. Mol. Biol. Rep. 1-12 (2021) doi:10.1007/s11033-020-06071-5.

50. Jørgensen, A. B., Frikke-Schmidt, R., Nordestgaard, B. G. \& Tybjærg-Hansen, A. Loss-offunction mutations in APOC3 and risk of ischemic vascular disease. N Engl J Med 371, 3241 (2014).

51. TG and HDL Working Group of the Exome Sequencing Project, N. H. Loss-of-function mutations in APOC3, triglycerides, and coronary disease. N. Engl. J. Med. 371, 22-31 (2014).

52. Cho, M. H. et al. Risk loci for chronic obstructive pulmonary disease: a genome-wide association study and meta-analysis. Lancet Respir. Med. 2, 214-225 (2014).

53. Pedroso, I. \& Breen, G. Gene set analysis and network analysis for genome-wide association studies. Cold Spring Harb. Protoc. 2011, pdb. top065581 (2011).

54. Pedroso, I. et al. Common genetic variants and gene-expression changes associated with bipolar disorder are over-represented in brain signaling pathway genes. Biol. Psychiatry 72, 311-317 (2012).

55. Frontera, C., Goicoechea, J., Ortín, J. \& Vives, E. Efficient algorithm for finding groundstates in the random field Ising model with an external field. J. Comput. Phys. 160, 117-125 (2000).

56. Hartmann, A. K. Ground-state structure of diluted antiferromagnets and random field systems. Phys. Stat. Mech. Its Appl. 248, 1-20 (1998).

57. Wang, L. et al. cisPath: an R/Bioconductor package for cloud users for visualization and management of functional protein interaction networks. in BMC systems biology vol. 9 1-6 (BioMed Central, 2015).

58. Reid, A. T. et al. Blocking Notch3 Signaling Abolishes MUC5AC Production in Airway Epithelial Cells from Individuals with Asthma. Am. J. Respir. Cell Mol. Biol. 62, 513-523 (2020).

59. Zhang, G. Expression and Prognostic Significance of BANF1 in Triple-Negative Breast Cancer. Cancer Manag. Res. 12, 145-150 (2020). 
60. Lin, Y.-Z. et al. Roundabout signaling pathway involved in the pathogenesis of COPD by integrative bioinformatics analysis. Int. J. Chron. Obstruct. Pulmon. Dis. 14, 2145-2162 (2019).

61. Kim, W. J. et al. Comprehensive Analysis of Transcriptome Sequencing Data in the Lung Tissues of COPD Subjects. Int. J. Genomics 2015, 1-9 (2015).

62. Radom-Aizik, S. et al. Effects of exercise training on quadriceps muscle gene expression in chronic obstructive pulmonary disease. J. Appl. Physiol. 102, 1976-1984 (2007).

63. Liu, X.-D. et al. Integrated analysis of lncRNA-mRNA co-expression networks in the $\alpha$ particle induced carcinogenesis of human branchial epithelial cells. Int. J. Radiat. Biol. 95, 144-155 (2019).

64. Mairinger, F. D. et al. Gene Expression Analysis of the 26S Proteasome Subunit PSMB4 Reveals Significant Upregulation, Different Expression and Association with Proliferation in Human Pulmonary Neuroendocrine Tumours. J. Cancer 5, 646-654 (2014).

65. Zhu, Y., Zhou, A. \& Li, Q. Whole transcriptome analyis of human lung tissue to identify COPD-associated genes. Genomics 112, 3135-3141 (2020).

66. Clark, H. et al. Differential associations of allergic disease genetic variants with developmental profiles of eczema, wheeze and rhinitis. Clin. Exp. Allergy 49, 1475-1486 (2019).

67. Wu, X., Sun, X., Chen, C., Bai, C. \& Wang, X. Dynamic gene expressions of peripheral blood mononuclear cells in patients with acute exacerbation of chronic obstructive pulmonary disease: a preliminary study. Crit. Care 18, 508 (2014).

68. $\mathrm{Xu}, \mathrm{S}$. et al. The association of APOC4 polymorphisms with premature coronary artery disease in a Chinese Han population. Lipids Health Dis. 14, 63 (2015).

69. Gerber, Y., Goldbourt, U., Cohen, H. \& Harats, D. Association between serum apolipoprotein CII concentration and coronary heart disease. Prev. Med. 35, 42-47 (2002).

70. Castro, A. et al. APOH is increased in the plasma and liver of type 2 diabetic patients with metabolic syndrome. Atherosclerosis 209, 201-205 (2010).

71. Genetic Determinants of Cardiovascular Disease Risk in Familial Hypercholesterolemia. https://www.ahajournals.org/doi/epub/10.1161/01.ATV.0000168909.44877.a7 doi:10.1161/01.ATV.0000168909.44877.a7.

72. Association of APOL1 Risk Alleles With Cardiovascular Disease in Blacks in the Million Veteran Program. https://www.ahajournals.org/doi/epub/10.1161/CIRCULATIONAHA.118.036589 doi:10.1161/CIRCULATIONAHA.118.036589.

73. Osada, J. Prenylcysteine oxidase 1 a pro-oxidant enzyme of low density lipoproteins. Front. Biosci. 23, 1020-1037 (2018).

74. Robins, S. J., Lyass, A., Brocia, R. W., Massaro, J. M. \& Vasan, R. S. Plasma lipid transfer proteins and cardiovascular disease. The Framingham Heart Study. Atherosclerosis 228, 230-236 (2013).

75. Baralla, A. et al. Plasma Clusterin and Lipid Profile: A Link with Aging and Cardiovascular Diseases in a Population with a Consistent Number of Centenarians. PLoS ONE 10, e0128029 (2015).

76. Packard, C. J. et al. Lipoprotein-Associated Phospholipase $\mathrm{A}_{2}$ as an Independent Predictor of Coronary Heart Disease. N. Engl. J. Med. 343, 1148-1155 (2000). 
77. Compromised LCAT Function Is Associated With Increased Atherosclerosis. https://www.ahajournals.org/doi/epub/10.1161/CIRCULATIONAHA.105.540427 doi:10.1161/CIRCULATIONAHA.105.540427.

78. Lara-Pezzi, E., Dopazo, A. \& Manzanares, M. Understanding cardiovascular disease: a journey through the genome (and what we found there). Dis. Model. Mech. 5, 434-443 (2012).

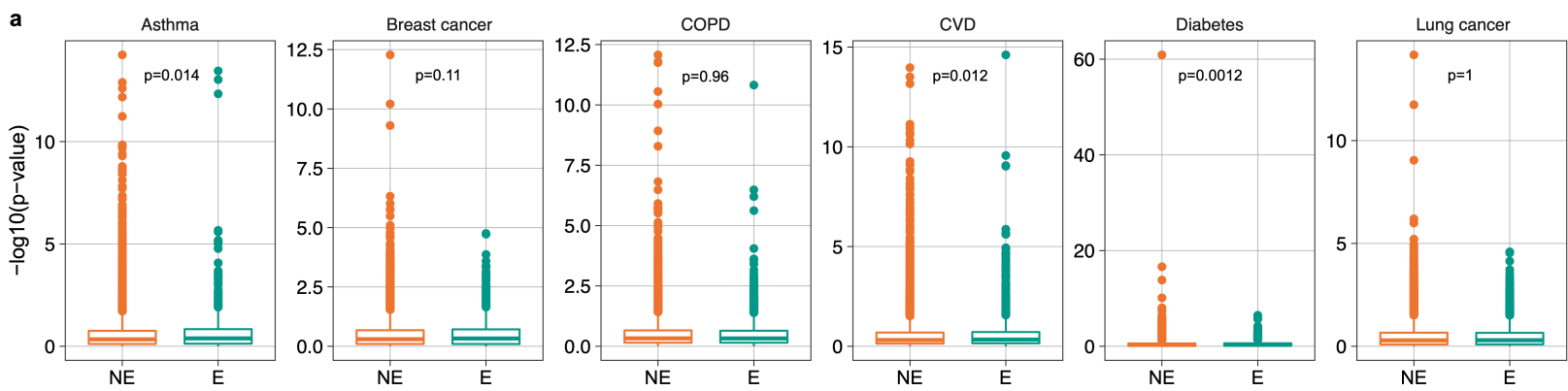

b STRING
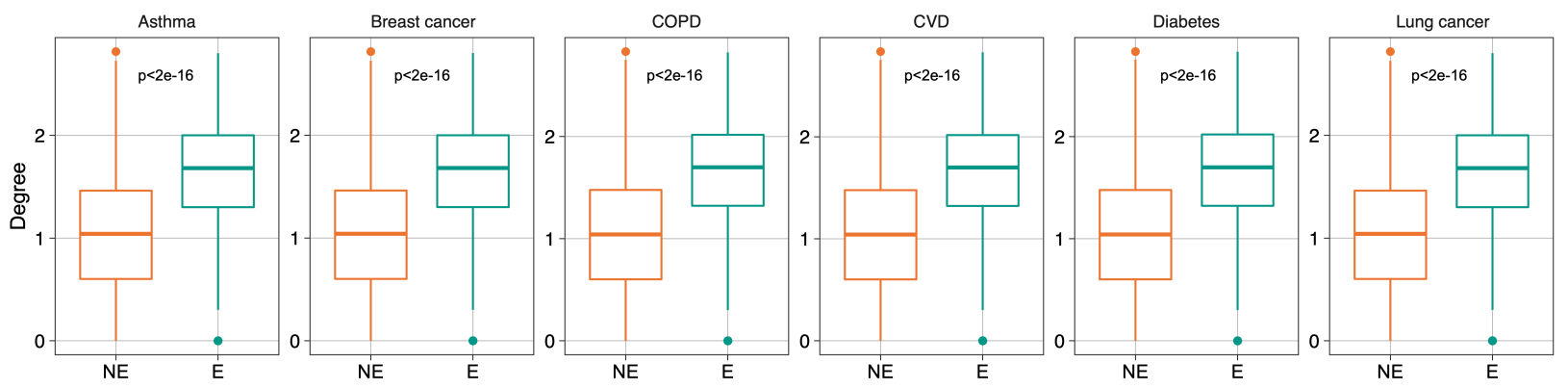

c iRefIndex

680

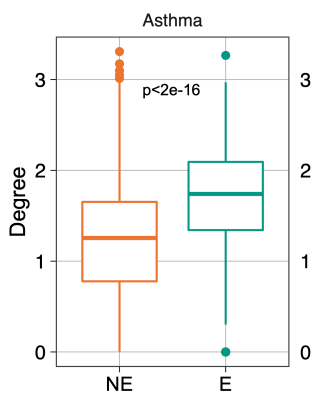

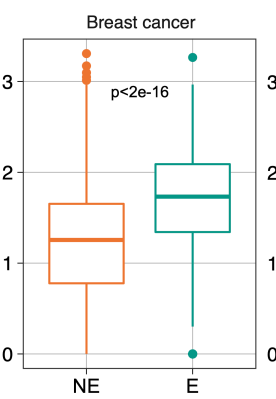
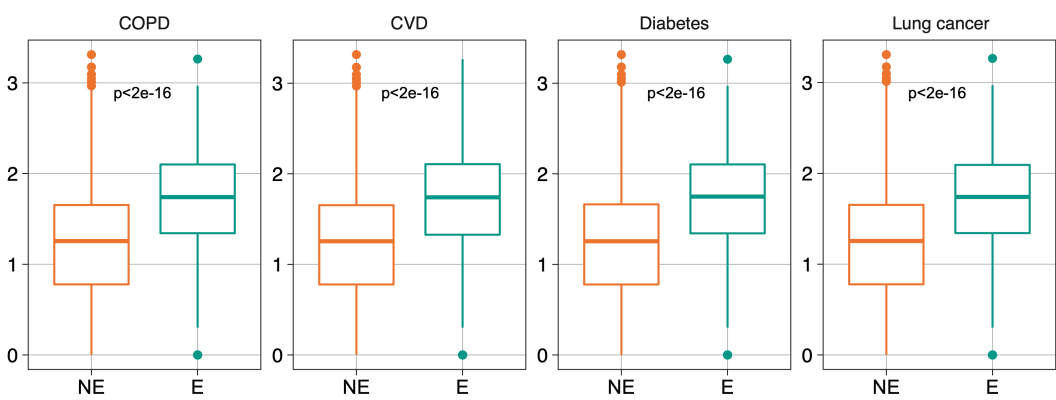

Figure S1: Correlation between gene essentiality, gene-wise p-value and degree. (a): Genewise p-value (in - $\log 10$ scale) distribution between essential and non-essential genes. (b): Gene degree (in $\log 10$ scale) distribution between essential and non-essential genes of STRING interactome. (c): Gene degree (in $\log 10$ scale) distribution between essential and non-essential genes of iRefIndex. Essential genes are defined as genes whose CS $<-0.1$ and corrected $\mathrm{p}<0.05$ in the KBM7 cell line. Significance level between essential and non-essential group were calculated using Wilcox test. 
a STRING
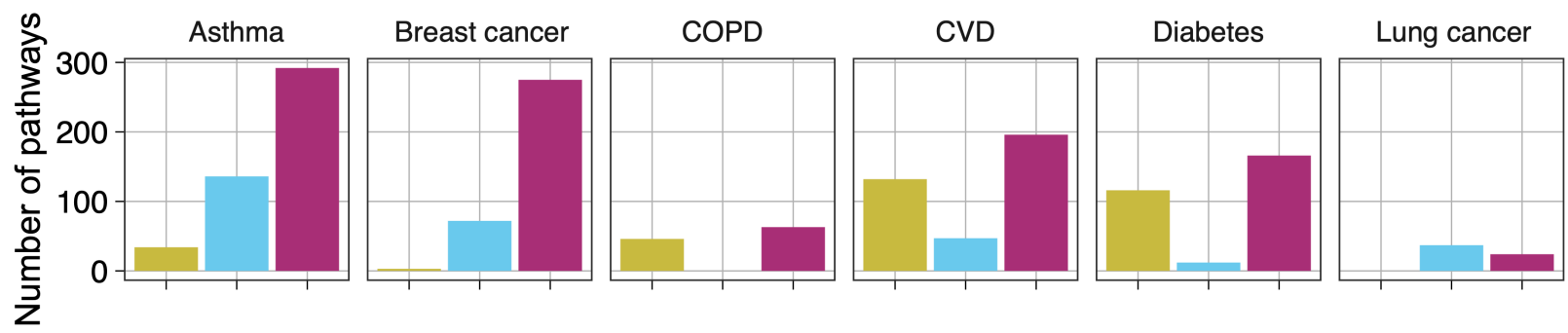

\section{b iReflndex}

693

694

695

696

697

698

699

700

701

702

703

704

705

706

707

708

709

710

711

712

713
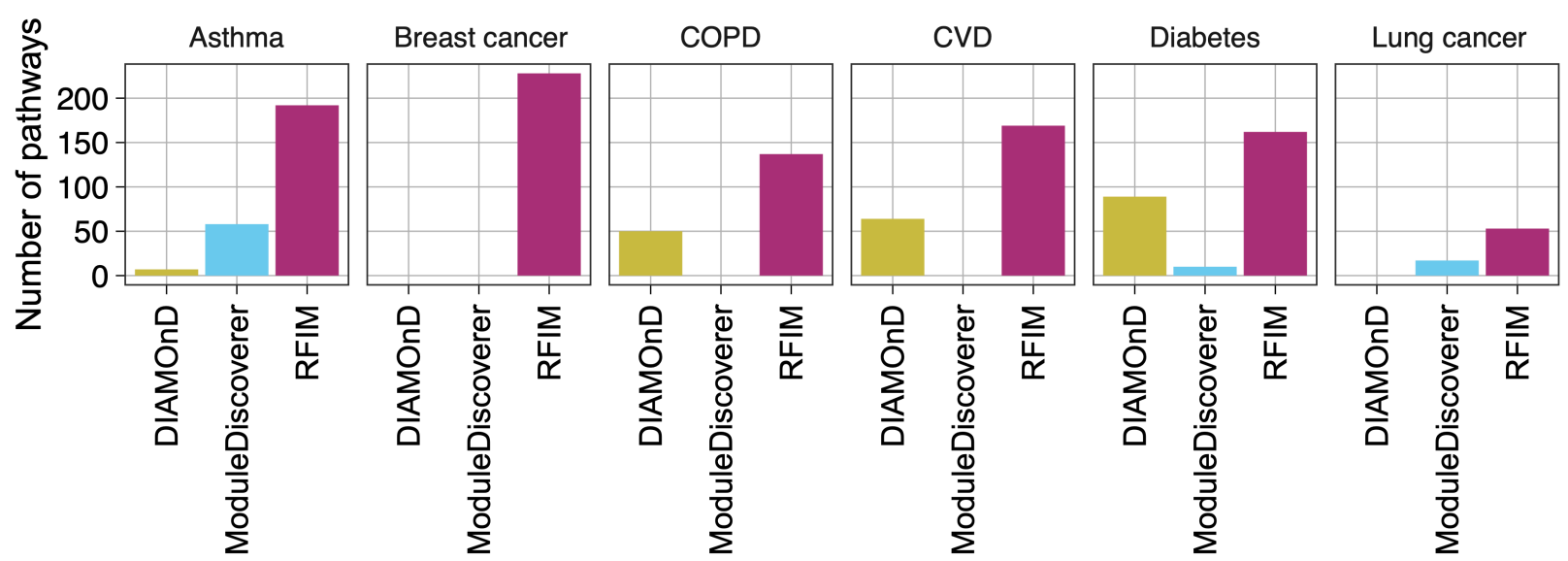

Figure S2: Enrichment analysis for genes in disease module detected from integrating the GWAS with four interactomes for each phenotype and method. We obtained the enriched pathways for disease genes using the ReactomePA package ${ }^{38}$ whose p-values are lower than 0.05 cutoff adjust by False Discovery Rate (FDR). Then, we extracted the disease-associated genes of each phenotype using the DisGeNET ${ }^{39}$ database and calculated the number of enriched pathways with at least two disease-associated genes. 

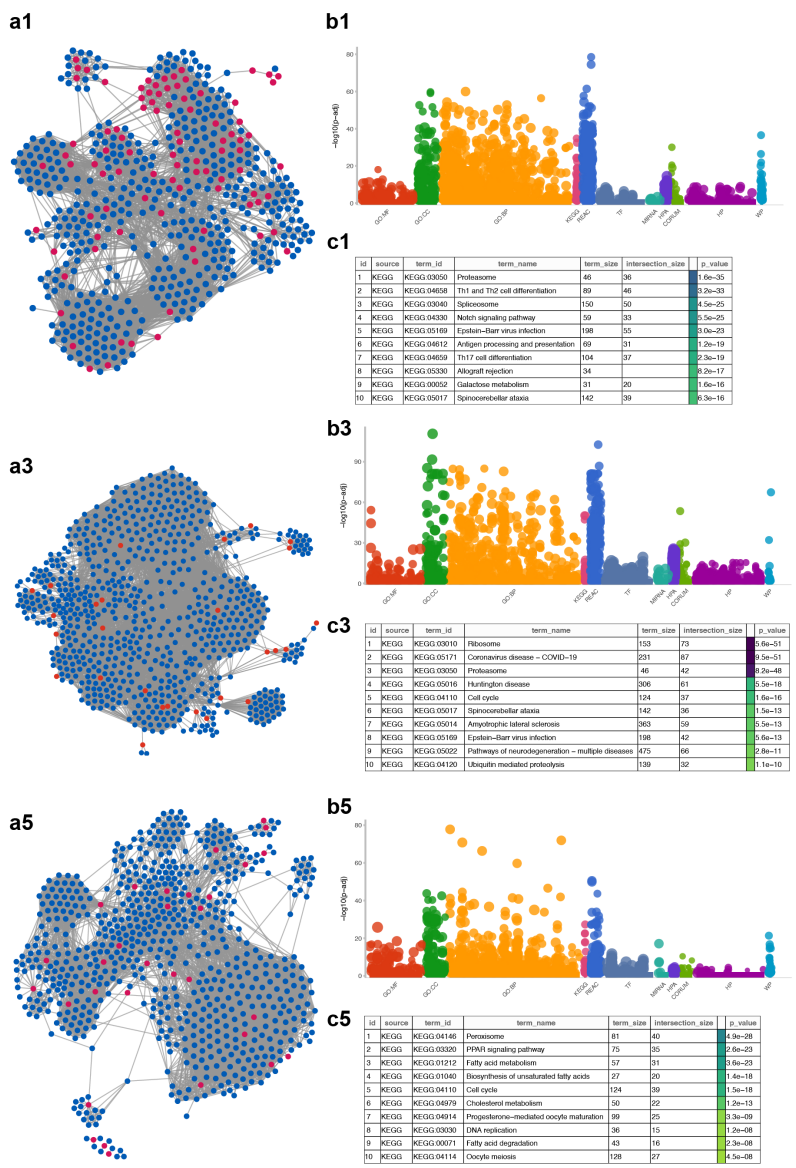
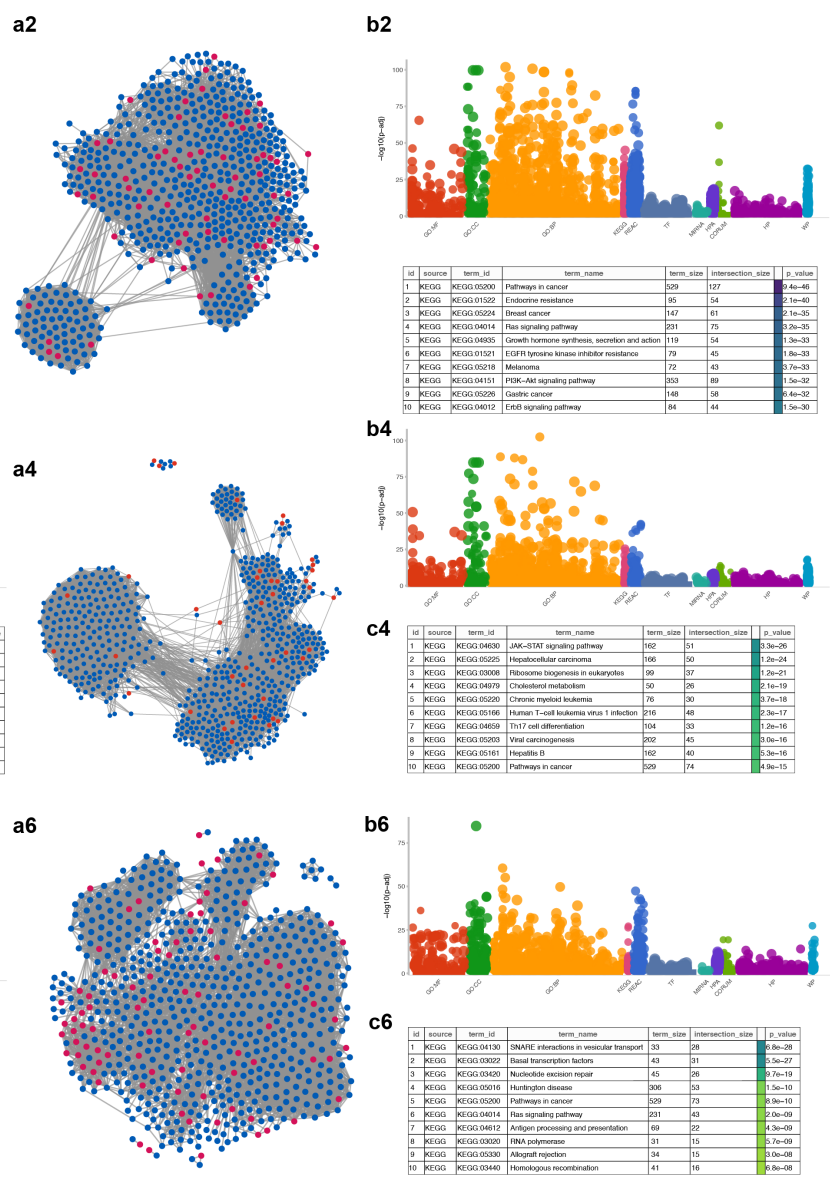

716

Figure S3: Subnetworks of genes in the disease module and enriched functional terms using STRING interactome. (a1-a6): Subnetwork of genes in disease modules of asthma, breast cancer, COPD, CVD, diabetes and lung cancer. Genes with p-values lower (higher) than 0.001 are colored with red (blue). (b1-b6): Enriched functional terms of genes in disease modules of asthma, breast cancer, COPD, CVD, diabetes and lung cancer using gprofier ${ }^{40}$. (c1-c6): Top-10 enriched KEGG pathways of genes in the disease modules of of asthma, breast cancer, COPD, CVD, diabetes and lung cancer. 

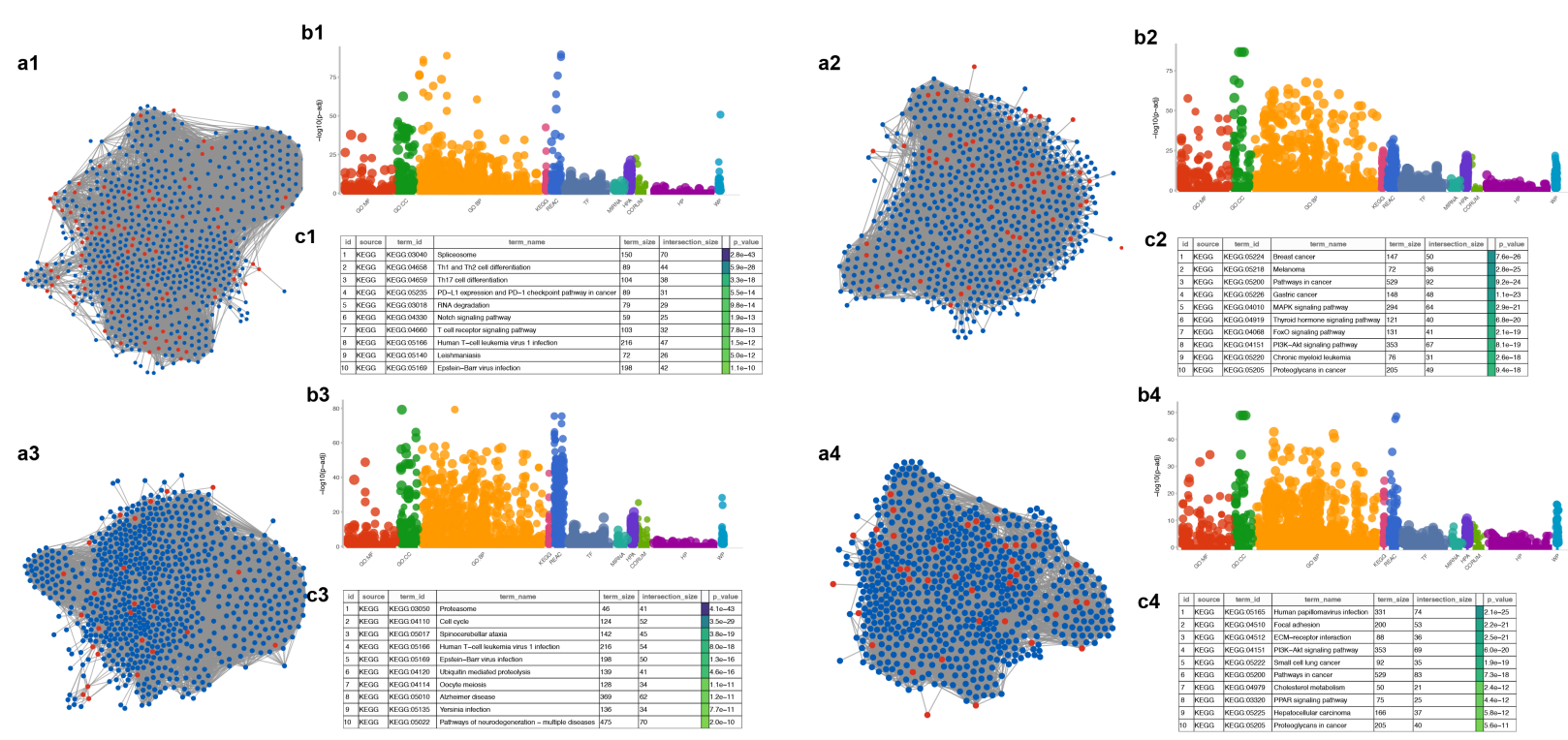

b4
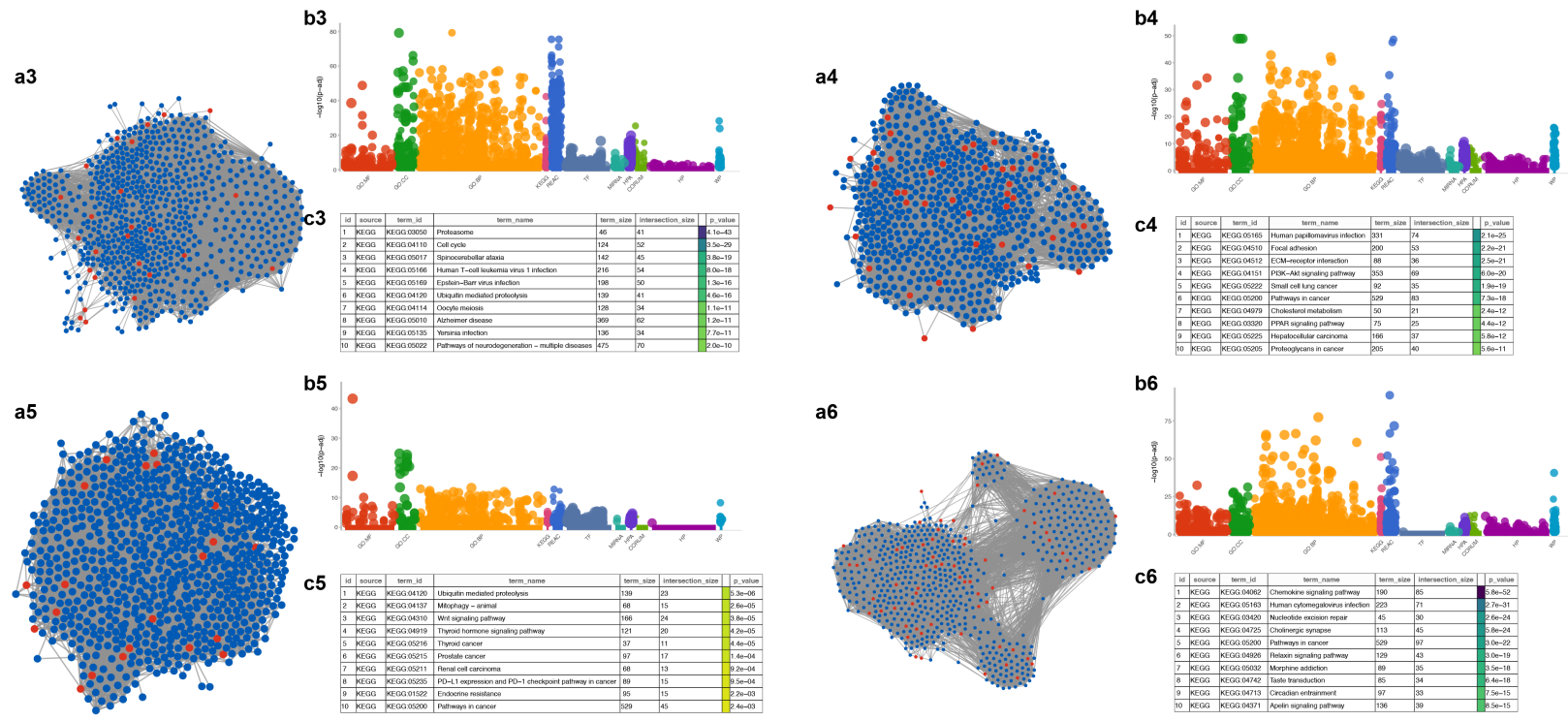

724

725

726

727

728

729

730

731

732

733

734

735

736

737

738

739

740

741

742

743

Figure S4: Subnetworks of genes in the disease module and enriched functional terms using iRefIndex interactome. (a1-a6): Subnetwork of genes in disease modules of asthma, breast cancer, COPD, CVD, diabetes and lung cancer. Genes with p-values lower (higher) than 0.001 are colored with red (blue). (b1-b6): Enriched functional terms of genes in disease modules of asthma, breast cancer, COPD, CVD, diabetes and lung cancer using gprofier2 ${ }^{40}$. (c1-c6): Top-10 enriched KEGG pathways of genes in the disease modules of asthma, breast cancer, COPD, CVD, diabetes and lung cancer. 